\title{
3 Französische und deutsche Kommunikationsformen im Kontext der Diskussion um ,Zivilisation` versus ,Kultur`
}

\subsection{Die Bedeutung mythisierender Konzepte wie ,Zivilisation und ,Kultur' in der deutsch-französischen Begegnung}

Am 3. März 2010 gab es im französischen Fernsehkanal France 3 eine Diskussion, die so in Deutschland mit einer entsprechenden Thematik niemals hätte stattfinden können: „Faut-il être nostalgique de la grandeur de la France?“ heißt der Titel der Sendung, in der sechs Historiker, Politikwissenschaftler und Journalisten ausgehend von den Thesen des Figaro-Korrespondenten Eric Zemmour aus dessen Buch Mélancholie française (2010) über die ehemalige ,grandeur de la France“ diskutierten. Zemmour geht davon aus, dass Frankreich als Nachfolger des Römischen Reichs auch die Größe der römischen Zivilisation geerbt und über die Jahrhunderte bewahrt hat. Er verfolgt die historischen Realisierungen dieses Narrativs eines französischen Sendungsbewusstseins seit dem frühmittelalterlichen Königtum von Clovis, welches sich in der Formel: ,La France n'est pas en Europe, elle est l'Europe‘ verdichten lässt. Mit dem Verlust der Schlacht von Waterloo 1815 sei Frankreich nach und nach dieser ,grandeur'verlustig gegangen.

Jeder, der in Frankreich im ökonomischen Sektor aktiv ist oder mit Bildungsbzw. politischen Einrichtungen zu tun hat, wird in der ein oder anderen Form mit dem französischen Bewusstsein ehemaliger ,grandeur' sowie der Klage über deren Verlust in der globalisierten Gegenwart konfrontiert. In seiner technikhistorischen Untersuchung über die Motive der Einführung unterschiedlicher Farbfernsehstandards in Deutschland und in Frankreich (PAL vs. SECAM) im Jahre 1967 hat Andreas Fickers gezeigt, inwieweit mythisierende Konzepte wie das Narrativ von der ,grandeur de la France' auf der einen Seite und jenes von der vermeintlich überlegenen deutschen Technologie unter dem Motto ,Made in Germany‘ auf der anderen Seite, die technischen, wirtschaftlichen, institutionellen und politischen Entscheidungen maßgeblich beeinflusst haben ${ }^{1}$. Es sind kulturelle Einstellungen und Mentalitäten, die seinerzeit zur Einführung von zwei unterschiedlichen Systemen statt eines einheitlichen technischen Standards in Europa geführt haben. Die Präferierung des SECAM-Systems in Frankreich, wo technische Entscheidun-

1 „Politique de la grandeur“ versus „Made in Germany“. Politische Kulturgeschichte der Technik am Beispiel der PAL-SECAM-Kontroverse (Pariser Historische Studien. 78), München, Oldenburg, 2007.

Ә Open Access. (C) 2021 Michael Bernsen, publiziert von De Gruyter (cc)BY-NC-ND Dieses Werk ist lizenziert unter der Creative Commons Attribution 4.0 Lizenz.

https://doi.org/10.1515/9783110758009-003 
gen allzu häufig zusammen mit wirtschaftlichen und politischen Entscheidungen auf nationaler Ebene gekoppelt werden, spielte in Charles de Gaulles Konzept der ,politique de la grandeur' eine entscheidende Rolle. Der Versuch, den französischen technischen Standard auf der europäischen Ebene durchzusetzen, war ein Versuch der Rückgewinnung historisch verlorener ,grandeur' des Landes, zumal es gelang, Russland als Abnehmer der Technologie mit ins Boot zu holen, welches wiederum auf die DDR Einfluss ausüben und damit das deutsche PAL-System unter Druck setzen konnte ${ }^{2}$. Wie Fickers anhand sogfältig ausgewerteter Archivmaterialien zeigen kann, ist das Narrativ der ,grandeur` erklärtermaßen die Grundlage der Bemühungen der französischen Seite. In einer geheimen Protokollnotiz des damaligen Kabinettsmitglieds Philippe Olivier vom 2. September 1965 heißt es nämlich :

C'est au mois de décembre 1964 que le gouvernement français décide de faire de l'adoption du procédé SECAM par les pays européens, sans exclure bien entendu les autres, une affaire d'intérêt national. [...] Le SECAM est un procédé de haute qualité, il intéresse une industrie pilote de notre économie, son adoption par le continent européen tout entier constituerait un succès industriel et technique certes, mais essentiellement politique de première grandeur pour notre pays. ${ }^{3}$

Ein Blick auf die Historie dieses Narrativs und seines Umfeldes entpuppt sich als besonders aufschlussreich, zumal dies die Gelegenheit gibt, zwei Schlüsselbegriffe dieser Untersuchung einzuführen und deren historischen Bedeutungswandel nachzuvollziehen. Dabei handelt es sich um ein interkulturelles Phänomen der deutsch-französischen Begegnung: Die Vorstellung von der ,grandeur de la France' resultiert unmittelbar aus der Anschauung von der ,civilisation française', die Frankreich in Europa spätestens seit dem 17. Jahrhundert eine herausragende Stellung beschert hat. Eine wesentliche Äußerungsform dieser Zivilisation ist die indirekte Kommunikation. Im deutschsprachigen Raum wird der französischen Zivilisation seit dem 18. Jahrhundert der Begriff der ,Kultur' entgegengengesetzt und die deutsche Erzählung von der technischen Überlegenheit, des ,Made in Germany', geht auf die alte Besinnung der Deutschen auf ihre Kultur zurück, ist doch die Technik als Beherrschung der Natur eine herausragende Form der Kultur. Die Abgrenzung der ,Zivilisation“ von der ,Kultur' wird gut 200 Jahre lang in immer neuen Schattierungen vorgenommen und hat auf diese Weise erhebliche Auswirkungen auf die mentalen Einstellungen der Bürger beider Länder nach sich gezogen.

2 Vgl. S. 33 und S. 346.

3 S. 254; Hervorhebung von Vf. 


\subsection{Französische, Zivilisation`}

Die Zivilisation, abgeleitet vom lat. ,civitas', ist die „Verbegrifflichung der Stadtkultur als Folge der Arbeitsteilung Stadt-Land“ ${ }^{4}$. Die mit der Zivilisation verbundenen Werte wie die ,urbanitas' oder die ,politesse‘ (vom griech. ,polis') macht sich der ,citoyen` zu eigen, in Frankreich hauptsächlich die Oberschicht von Paris, die sich über diese Anschauungen von der Provinz und vom Land abgrenzt ${ }^{5}$. Der Begriff ,Zivilisation' taucht zum ersten Mal im Jahr 1759 in Victor Riquetti de Mirabeaus Abhandlung L'ami des hommes, ou traité de la population auf ${ }^{6}$. Der französische Philosoph Condorcet definiert ,civilisation' dann wenig später in seinem Esquisse d'un tableau historique des progrès de l'esprit humain (1794-1795) als Gegensatz zur Barbarei - bei ihm „peuplades sauvages“ genannt ${ }^{7}$ - und sieht seine Epoche der Aufklärung als höchste Stufe der Zivilisation. Das nationale Bewusstsein Frankreichs definiert sich vornehmlich über diesen Begriff der ,Zivilisation'.

Die Entstehung der Zivilisation hat insbesondere Norbert Elias 1939 in seinem epochemachenden Buch Über den Prozess der Zivilisation analysiert. Am Beispiel der französischen Entwicklung der Hofgesellschaften bis hin zum absolutistischen Zentralstaat des 17. Jahrhunderts beschreibt Elias unter dem Stichwort der ,Zivilisierung، einen langfristigen Wandel der Persönlichkeitsstrukturen, der wiederum von der Entwicklung der Sozialstrukturen abhängt. Der technische Fortschritt und die Differenzierung sowie der zunehmende Konkurrenzkampf zwischen den Menschen bringen eine Zentralisierung in Gestalt des staatlichen Gewaltmonopols mit sich. Dadurch entstehen Interaktionsketten ${ }^{8}$, in die die Menschen eingebunden sind. Diese Bindungen führen wiederum zu einer profun-

4 Hans-Otto Dill, „Kultur vs. Zivilisation - Genesis zweier anthropologischer Grundbegriffe”, Sitzungsberichte der Leibniz-Sozietät der Wissenschaften zu Berlin Bd. 111/2011, S. 131-158, hier: S. 137.

5 Vgl. ebd.

6 Mirabeau führt die Zivilisation noch auf den heilsamen Einfluss der Religion zurück: „La Religion est sans contredit le premier \& le plus utile frein de l'humanité; c'est le premier ressort de la civilisation: elle nous prêche, \& et nous rappele sans cesse la confraternité, adoucit notre cœur, éleve notre esprit, flatte \& dirige notre imagination, en étendant le champ des récompenses \& des avantages dans un territoir sans bornes, \& nous intéresse à la fortune d'autrui [...]“ (L'ami des hommes ou traité de la population, 2 Teile, Avignon 1756-1760, Teil 1, Kap. 8, S. 192).

7 Marie Jean Antoine Nicolas Caritat, Marquis de Condorcet, Esquisse d'un tableau historique des progrès de l'esprit humain, hrsg. von Yvon Belaval (Collections des textes philosophiques), Paris, Vrin, 1970 (11794-1795), S. 43.

8 Norbert Elias, Über den Prozess der Zivilisation. Soziogenetische und psychogenetische Untersuchungen (suhrkamp taschenbuch wissenschaft. 158. 159), 2. Bde., Frankfurt a. M., Suhrkamp, 1976, Bd. 2, bes. S. 325 f. 
den Veränderung der menschlichen Persönlichkeit: Sie zwingen den Einzelnen zur Kontrolle seiner Affekte und zur Langsicht über die Folgen seiner Handlungen. Spontane emotionale Impulse werden zurückgedrängt und die Handlungen des Menschen zunehmend einer rationalen Kontrolle unterworfen. Im Innern des Einzelnen - so Elias - entsteht ein „Zaun von schweren Ängsten“9, der ihn vor gefährlichen Auswirkungen spontaner impulsiver Handlungen bewahrt. Die Scham- und Peinlichkeitsschwellen des Einzelnen rücken vor; seine Gewaltbereitschaft weicht einer Zurückhaltung. Der Einzelne entwickelt zugleich psychologische Fähigkeiten, die es ihm ermöglichen, interessenbedingte Affekte des Gegenübers sowie rationale Verhaltensmuster, die die Interessen kontrollieren, aber auch verdecken, zu erkennen. Insbesondere beim französischen Adel, der im 17. Jahrhundert den Status des Territorialfürsten verliert und am Hof von Versailles zusammengezogen wird, kann Elias diesen Prozess der Zivilisierung in Gestalt einer Verhöflichung der ehemaligen Krieger (,noblesse d'épée') beobachten. Die Zivilisierung bringt eine Verfeinerung aller Sitten und Manieren mit sich; sie ersetzt direktes, impulsives Handeln durch die ,courtoisie‘. Diese Zivilisierung der Persönlichkeit ist bei Elias eng verbunden mit der Zentralisierung der politischen Macht in ihrer herausragenden Gestalt des absolutistischen Machtstaats, wie er aus der Staatstheorie des Thomas Hobbes sowie aus der Herrschaft Ludwigs XIV. bekannt ist. Sie wird nicht nur zum Vorbild für die europäische Aristokratie; sie gilt auch dem Bürgertum des darauffolgenden 18. Jahrhundert als beispielhaftes Verhalten.

In seinen die Zivilisationstheorie von Elias differenzierenden Betrachtungen geht der Soziologe Clemens Albrecht allerdings davon aus, dass die in Frankreich entstehende Zivilisation, die, verbunden mit der Beherrschung der französischen Sprache, in allen bedeutenden europäischen Ländern bis zum Beginn des 19. Jahrhunderts durch die Oberschichten imitiert wird, ursächlich nicht primär aus der Machtpolitik des absolutistischen Staates entstanden ist. Nicht die Politik führt zur ,zivilisierenden Leitkultur“10 bzw. zur „repräsentativen Kultur“111, sondern die Gesellschaft der literarischen und philosophischen Salons der Stadt Paris, in denen sich unterschiedliche gesellschaftliche und soziale Gruppierungen zusam-

\footnotetext{
9 S. 317.

10 Clemens Albrecht, „,Kulturelle Hegemonie ohne Machtpolitik. Über die Repräsentativität der französischen Salonkultur“, in: Robert Simanowski/Horst Turk/Thomas Schmidt (Hrsg.), Europa - ein Salon? Beiträge zur „Internationalität des literarischen Salons (Veröffentlichungen aus dem Sonderforschungsbereich 529 „Internationalität nationaler Literaturen”. 6), Göttingen, Wallstein, 1999, S. 66-80. Vgl. auch ders., Zivilisation und Gesellschaft. Bürgerliche Kultur in Frankreich, München, Fink, 1999. S. 71.
}

11 S. 67. 
menfinden. In den Salons, die die Betroffenen in freier Assoziation besuchen, ist man gezwungen, sich den Vorgaben der ,civilité, die den jeweiligen Salon auszeichnen, zu unterwerfen. Oberstes Ziel ist die Erlangung von Ruhm, zu dessen Gewinn die Protagonisten die Normen und Werte des Salons verinnerlichen. Daraus ergebe sich - so Albrecht - eine größere „Homogenität des sozialen Handelns durch Verinnerlichung des Herrschaftsanspruchs als Kulturideal“ 12 als am Hof, an dem erheblich heterogenere Interessenlagen aufeinandertreffen. $\mathrm{Zu}$ diesem Kulturideal gehören, dies zeigen die weiter unten behandelten Verhaltenstraktate, ganz wesentlich die indirekte Kommunikation und die Kunst des Impliziten. Aus der zivilisierenden Herrschaft der Salondamen wird dann im 18. Jahrhundert die Herrschaft der öffentlichen Meinung.

Nach der französischen Revolution werden die Werte der Zivilisation in Frankreich als universell angesehen. Sie werden schon in der Aufklärung zentraler Bestandteil eines Narrativs, welches unter dem Namen ,mission civilisatrice“ auf ihren Export in andere Länder Europas und später in die Kolonien drängt. So hatte Marie Jean Antoine Nicolas de Caritat, Marquis de Condorcet, unmittelbar nach der Französischen Revolution davon gesprochen, dass zahlreiche außereuropäische Länder geradezu darauf warten würden, von den Europäern (gemeint sind in erster Linie die Franzosen) ,zivilisiert“ zu werden:

Ces vastes pays [...] semblent n'attendre, pour se civiliser, que d'en recevoir de nous les moyens, et de trouver des frères dans les Européens, pour devenir leurs amis et leurs disciples $[\ldots]^{13}$

Napoleon versucht dann die Errungenschaften der Revolution und die universellen Werte der Zivilisation nicht nur innerhalb des europäischen Kontinents zu verbreiten, sondern exportiert diese mit seiner Expedition nach Ägypten auch auf den afrikanischen Kontinent und in der Folge darüber hinaus in die Welt. Durch die Expedition nach Ägypten und die sich anschließende Besetzung Algeriens im Jahre 1830 entsteht eine systematische, imperiale Form des Kolonialismus, die insbesondere in der Dritten Republik zwischen 1870 und 1940 zur Erzählung von der ,mission civilisatrice“ verdichtet wird. Ein beliebtes Anschauungsmuster dieses Narrativs ist der Verweis auf die Römer, die Frankreich seinerzeit die glücksbringende Zivilisation gebracht hätten, welche die Nation ihrerseits nun den noch nicht zivilisierten ,Naturvölkern‘ der Welt spende. Diese Erzählung bedingt dann die in den Principes de Colonisation et de Legislation Coloniale des Juristen Arthur Girault 1895 formulierte Politik der ,assimilation': In den Kolonien soll über

12 S. 78.

13 Esquisse d'un tableau historique, S. 197 (Dixième époque). 
eine entsprechende Schulausbildung und die Loslösung der dortigen Eliten aus gewachsenen sozialen Bindungen eine europäisierte Führungsriege geschaffen werden. Die ,mission civilisatrice‘ ist gepaart mit jenem Narrativ, das bis heute im französischen Bewusstsein eine entscheidende Rolle spielt: eben jener Erzählung von der ,grandeur de la nation'. Seit dem Mittelalter, genauer seit der König Clovis es als seine ureigene Sendung ansah, das römische Reich zu erneuern, ist in Frankreich die Vorstellung dieses Sendungsbewusstseins vorhanden ${ }^{14}$. Gemäß der Geschichtsauffassung ,Große Männer machen Geschichte' macht sich diese Erzählung insbesondere an solchen ,grands hommes' wie Louis XIV fest, den Charles Perrault in seinem Gedicht Le siècle de Louis le Grand (1687) schon zu Lebzeiten entsprechend etikettiert, dann an Napoleon und schließlich an Charles de Gaulle, der bekanntermaßen in seinen Kriegsmemoiren behauptet, ohne,grandeur‘ wäre Frankreich nicht Frankreich (,[...] la France ne peut être la France sans la grandeur"15). Das Konzept der Zivilisation ist somit in Frankreich ein fundamentaler Baustein des nationalen Bewusstseins.

Die Unterscheidung der Begriffe ,Zivilisation` und ,Kultur` ist bereits in Elias Theorie grundlegend, zumal er die Historie beider Begriffe nachzuzeichnen sucht. Während man in Frankreich eher stolz von den Fortschritten der Zivilisation spricht und diese dem universalistischen Denken der Aufklärung gemäß allen Völkern als verbindliches Modell anpreist, wird die Vorstellung von der ,Zivilisation' in Deutschland insbesondere mit der französischen Aristokratie, dann aber auch mit der einheimischen Aristokratie in Verbindung gebracht, soweit diese die französische nachahmt. Gegen Ende des 18. Jahrhundert gerät die Nachahmung Frankreichs und dessen Zivilisation in Deutschland zunehmend in Verruf. Der Zivilisation setzt man in Deutschland die Bewahrung der eigenen Kultur entgegen. Dies hat wiederum ganz maßgeblich mit dem französischen Kommunikationsstil des indirekten Sprechens zu tun.

\subsection{Deutsche, Kultur}

Der Begriff, Kultur' geht auf das lat. ,colere‘, ,bebauen, pflegen' zurück. Er bezeichnet grundsätzlich das, was der Mensch hervorbringt. Kultur ist eine Form der Bewältigung der Natur. Mittels der Kultur hebt sich der Mensch von der ,Natur ‘ ab, die gegeben ist. Von seiner Etymologie her gesehen hat der Begriff ,Kultur' daher konnotativ stets einen engen Bezug zum Ackerbau und zur Landwirtschaft. Im

14 Vgl. dazu Myriam Yardeni, Enquêtes sur l'identité de la „Nation France“. De la Renaissance aux Lumières, Seyssel, Champ Vallon, 2005, Kap. 8, S. 112-125.

15 Charles de Gaulle, Mémoires de guerre et mémoires d'espoir, Paris, Plon, 2016, S. 10. 
Kontext der Fragestellung dieses Buchs ist vor allem eine weitergehende Definition des sehr vielgestaltigen Kulturbegriffs von Interesse: Kultur als dasjenige, was eine bestimmte Gruppe von Menschen miteinander verbindet. Über den Bereich der technischen Bewältigung der Natur hinaus liefert die Kultur einer Gruppe Orientierungen und sichert damit deren Identität. In der Kultur dieser Gruppe werden gemeinsame Werte und Normen festgelegt und das für die Gruppe typische Verhalten definiert. Kultur ist dadurch stark traditionsverhaftet. Kulturelle Verhaltensweisen haben ihren festen Bestand in der Geschichte der Gruppe und können durch historische Betrachtungen in ihren Dimensionen beschrieben werden.

Folgt man Immanuel Kant, so erfasst der Begriff ,Zivilisation“ eher äußerliche Verhaltensweisen, die sich vor allem auf die Manieren im gegenseitigen Umgang der Menschen in der Gemeinschaft beziehen. Kant spricht von einer „äußeren Anständigkeit“, die er als bloße „Civilisirung [...] bis zum Überlästigen“ abtut. Dieser Zivilisierung fehlt Kant die innere moralische Integrität, die impliziert, dass die Menschen ihr Handeln und Tun auf gute Zwecke ausrichten. „Zur Cultur“, so Kant, gehört demnach die „Idee der Moralität“16. Die Zivilisation wird auf diese Weise als taktvolle Form des Handelns von der Kultur unterschieden, die als eine moralische, ja nahezu religiös fundierte, Humanität angesehen wird.

Diese starke Abgrenzung des Kulturbegriffs vom Begriff der Zivilisation in Deutschland ist ein historisches Phänomen deutsch-französischer Interkulturalität: Mit der Betonung der Kultur in Deutschland setzt man sich von der ,civilité‘ Frankreichs ab, die man mit dem aristokratischen Verhalten bei Hof und in den Salons und dessen vor allem großstädtische Prägung durch die zentrale Ausrichtung auf die Metropole Paris in Verbindung bringt. Die französische Zivilisation gilt Teilen der deutschen bürgerlichen Spätaufklärung als affektierte, degenerierte und oberflächliche Form menschlichen Zusammenlebens, der man die Natürlichkeit der eigenen, erheblich stärker regional und ländlich geprägten Lebensweise mit ihrer gewachsenen, tief verankerten Kultur entgegenstellt:

[Kultur avanciert] zu einem Kampfbegriff der deutschen Aufklärung gegenüber dem Sittenkodex des Adels, der sich im politisch zersplitterten Deutschland vor allem an den Konventionen des übermächtigen französischen Hofes orientiert hatte. Im Gegensatzpaar Kultur und ,Civilité‘ formuliert sich somit nicht nur eine standespolitische, sondern auch eine nationale Konfrontation zwischen Frankreich und den deutschsprachigen Ländern. ${ }^{17}$

16 Immanuel Kant, Idee zu einer allgemeinen Geschichte in weltbürgerlicher Absicht (1784). in: I. K., Gesammelte Schriften, hrsg. von der Königlich Preußischen Akademie der Wissenschaften, 28 Bde., Berlin, Georg Reimer, 1900-1955, Bd. 8, S. 15-32, hier: S. 26.

17 Gabriele Klein, „Kultur“, in: Hermann Korte/Bernhard Schäfers (Hrsg.), Einführung in die Hauptbegriffe der Soziologie (Einführungskurs Soziologie), Wiesbaden, Verlag für Sozialwissenschaften, ${ }^{8} 2010$ ( $\left.{ }^{1} 2003\right)$, S. 235-256, hier: S. 236. 
Die zunächst doppelte soziale Abgrenzung (gegenüber der Aristokratie sowie der städtischen Lebensweise) geht im Laufe des 18. Jahrhunderts in eine nationale Abgrenzung über, wodurch die antithetischen Anschauungen von der (französischen) Zivilisation und der (deutschen) Kultur eine erhebliche Bedeutungserweiterung und Bedeutungsverlagerung erfahren ${ }^{18}$. Im deutschen Begriff ,Kultur' spiegelt sich - so Elias - das Selbstbewusstsein einer aufkommenden mittelständischen Intelligenzschicht, die weitgehend jenseits der in Deutschland stark zersplitterten höfischen Gesellschaften angesiedelt is $\mathrm{t}^{19}$. Diese Schicht wendet sich im Namen einer bei den Deutschen angeblich verinnerlichten Tugendhaftigkeit und Natürlichkeit gegen die Oberflächlichkeit und Äußerlichkeit der französischen Zivilisation. Dies lässt sich bereits am - von Elias nicht beachteten - Eintrag „Teutschland, Deutschland, Teutsches-Reich“ in Johann Heinrich Zedlers Universal-Lexicon ablesen, wo es von den Deutschen heißt:

Von ihren Neigungen zu gedencken, so sind sie, da sie was Cholerisches haben, treu, redlich, daraus sie sich eine Ehre machen, und Hinterlist als etwas schädliches ansehen, daher man auch ihren Muth und Tapfferkeit herzuleiten hat, massen selbige ein Kennzeichen der Cholericorum ist. ${ }^{20}$

Die Deutschen sind, so Zedler, bar jeder Hinterlist, was deutlich gegen die in Frankreich in höfischen Kreisen sowie in den Salons praktizierte ,dissimulatio der Affekte und Gedanken gerichtet ist. Es ist der französische Kommunikationsstil des indirekten Sprechens, auf den die Beschreibung der Deutschen zielt. Die Deutschen sind naturverbunden, was sich im cholerischen Grundzug als einer natürlichen, unkontrollierten Gefühlsreaktion äußert. Dabei sind sie jedoch dem Grundsatz der Treue und Redlichkeit verpflichtet. Diese Definition eines deutschen, nahezu schon nationalen Selbstbewusstseins - „daraus sie sich eine Ehre machen“, heißt es - wird gegen französisches Verhalten gestellt, von dem der weitere Verlauf des Artikels berichtet. Darin beklagt Zedler die deutsche Nachahmung Frankreichs, die vor allem durch die Einwanderung der „Reformierten“ aus Frankreich gefördert werde. „Die Französische Nation ist veränderlich, dies ist eine weltbekannte Sache“21 - heißt es, womit die vermeintliche Oberflächlichkeit der französischen Zivilisation thematisiert wird. Die französische Zivilisation

18 S. $36 \mathrm{ff}$.

19 Über den Prozess der Zivilisation, S. 31.

20 Johann Heinrich Zedler, Grosses vollständiges Universal-Lexicon aller Wissenschaften und Künste, 64 Bde., 4 Suppl.-Bde., Leipzig/Halle, Zedler, 1732-1754; Nachdruck: Graz 1962, Bd. 43, Sp. 273-295, hier: Sp. 292.

21 Sp. 293. 
bestehe vor allem in flüchtigen Modeerscheinungen wie „Kleidungen [...] in der Art zu speisen, in Meubeln, in Equipagen [... ]“22.

Deutsches Selbstbewusstsein entsteht demnach über einen Kulturbegriff, der vornehmlich aus der Abgrenzung von der französischen Zivilisation resultiert. Der Kulturbegriff ist ausgrenzend: Er zielt auf die Fixierung der Anschauungen des eigenen Verhaltenskodexes in der von äußeren Einflüssen abgeschotteten Gemeinschaft. Charakteristikum des Zivilisationsbegriffes ist dagegen das Prozesshafte, welches vor allem in der Begriffsnuance der ,Zivilisierung' zum Ausdruck kommt ${ }^{23}$ und welches dann im 19. Jahrhundert während des Kolonialismus virulent wird, wo die nationale Grenzen überschreitende Zivilisation als universalistischer Wert von Frankreich in andere Länder exportiert wird, um der dortigen ,Barbarei' Herr zu werden. Inwieweit sich ein deutsches kulturelles Selbstverständnis an der Zivilisation Frankreichs abarbeitet, zeigt auch die Entstehung eines deutschen nationalen Gründungsmythos von Hermann dem Cherusker, lat. Arminius.

Ausgelöst durch die wiederentdeckten Schriften des Tacitus ${ }^{24}$ wird Arminius im Verlauf des 16. Jahrhunderts durch Ulrich von Hutten und Philipp Melanchthon zum Befreier Germaniens von der römischen Vorherrschaft stilisiert, ein Konzept, dass den Bemühungen der protestantischen Abkehr von der römischen Kirche dienstbar gemacht wird. Bereits in einem der ersten Rezeptionsbeispiele dieser Diskussion in Deutschland, in Daniel Caspar von Lohensteins monumentalem, ganz im Stil des heroisch-galanten Romans geschriebenen Opus Großmüthiger Feldherr Arminius von 1689-1690, wird deutlich, wie sich aus der Arminius-Geschichte ein nationaler Mythos herausbildet. Vor dem Hintergrund der offensiven Kriegspolitik Ludwigs des XIV. seit den siebziger Jahren des 17. Jahrhunderts dominiert bei Lohenstein der Topos von Arminius als dem Einiger der germanischen Stämme, wobei der Autor die Einigung der deutschen Reichsstände modellhaft vor Augen hat. Die protestantische Rezeption der Arminius-Figur als ,liberator Germaniae، lässt sich nicht nur gegen Rom als Sitz der katholischen Kirche wenden ${ }^{25}$, sondern auch gegen den französischen Absolutismus und die französische Zivilisation. Durch die Übermacht der französischen Aufklärung so-

22 Ebd. Elias, der den Artikel „Hof, Höflichkeit, Hofmann“ aus Zedler heranzieht, führt den Artikel „Teutschland“ nicht an, weil dieser seiner These vom Zurücktreten des sozialen und vom Hervortreten des nationalen Gegensatzes in der Antithese ,Zivilisation vs. Kultur‘ widerspricht.

23 Vgl. Über den Prozess der Zivilisation, S. 3 f.

24 Vgl. dessen Annales 2.88.2.

25 Vgl. dazu Thomas Borgstedt, Reichsidee und Liebesethik. Eine Rekonstruktion des Lohensteinschen Arminiusromans (Studien zur deutschen Literatur. 121), Tübingen, Max Niemeyer, 1992, S. 21. 
wie die sich an die Revolution anschließende Eroberung Europas durch Napoleon werden diese Reaktionen noch verstärkt. Im gedanklichen Feld der These Johann Gottfried Herders von der ,verspäteten Nation' schreibt Friedrich Gottlieb Klopstock seine Hermanntrilogie (1769-1787). Auf der Welle der Ossian'schen Bardengesänge führt er den Zeitgenossen die hohe Kultur der Germanen, die bei ihm zu Deutschen mutiert sind, vor Augen ${ }^{26}$. In Heinrich von Kleists 1808 geschriebener, jedoch erst 1821 gedruckter und noch viel später, im Jahr 1839, aufgeführter Hermannschlacht ist angesichts der Napoleonischen Besatzung der Gedanke der Einigung der Deutschen ganz zentral. Der deutsche Gründungsmythos von Hermann dem Cherusker, der sich der römischen Zivilisation entsagt hat und für die Einigung der germanischen Stämme steht, ist somit entstanden als ein Gegenpart gegen die französische Anschauung vom Erbe der römischen Zivilisation durch Frankreich sowie dem daraus resultierenden universalistischen Anspruch, diese Zivilisation in ganz Europa zu verbreiten ${ }^{27}$.

Die Fokussierung der deutschen Gründungsmythologie auf die HerrmannGeschichte führt in Frankreich zur Suche nach einer ebensolchen Gründungsfigur der Nation, die man bis dahin angesichts der universalistischen Ansprüche der im Lande entwickelten Zivilisation gar nicht vermisst hatte. Diese Figur findet sich in Vercingétorix, der seinerseits die Aufstände der Gallier gegen die Römer angeführt hatte. Über diese Erzählung ließ sich auch der Gründungsmythos von der politischen Zivilisierung Frankreichs verbreiten, da Vercingétorix die Schlacht von Alésia verloren hatte und die Gallier die römische Zivilisation übernehmen mussten. Da Vercingétorix jedoch in der Literatur und der Geschichtsschreibung bislang kaum Beachtung gefunden hatte, sieht sich die Académie Française gezwungen, 1865 eigens einen Concours de poésie zum Thema ,Vercingétorix‘ auszuschreiben. Beide Mythen haben in der zweiten Hälfte des 19. Jahrhunderts zur Errichtung monumentaler Denkmäler geführt, die sich als Zeichen nationalen Stolzes und nationaler Selbstbehauptung im Teutoburger Wald und in Alésia gegenüberstehen. Die beiden aufeinanderprallenden nationalen Mythen machen aber auch deutliche Unterschiede der Legitimierung des jeweiligen Nationalstaates sichtbar: Deutschland gibt sich auch in seinem Nationalmythos als Kulturnation zu erkennen, de-

26 Vgl. dazu Klaus Bemman, Arminius und die Deutschen, Essen, Magnus, 2002, S. 163 f.

27 Diese nationale deutsche Denkweise jenseits universeller zivilisatorischer Grundwerte zwingt auch Frankreich zur Suche nach nationalen Gründungsmythen. Aufgrund der sozialen Veränderungen nach der Französischen Revolution wird die sogenannte Germanenthese, wonach die französischen Herrscher von den germanischen Franken abstammen, durch die sogenannte Gallierthese, die Abstammung des französischen Volkes von den friedliebenden Galliern, ersetzt. Diese Auffassung wurde bereits im 18. Jahrhundert durch den Abbé Dubos vertreten und wird von den Historikern Amédée Thierry und Henri Martin im 19. Jahrhundert aufgegriffen. 
ren Volk sich über die Einheit von Sprache, Kultur und einer Freiheit definiert, die wesentlich auf dem negativen Wege der Erhaltung der nationalen Selbständigkeit gegenüber einem äußerlichen Aggressor - konkret von den Römern bis hin zu Napoleon - entstanden ist. Im Sinne Herders aus dem 57. der Briefe zu Beförderung der Humanität von 1794 sowie des deutschen Idealismus Schellingscher und Hegelscher Prägung verkörpert das Wirken des Arminius/Hermann demnach die Erhaltung eines politischen Raumes als Sprach- und Kulturraum. Demgegenüber ist das Verständnis von der Nation in Frankreich das einer Staatsnation. Diese ist historisch aus den sozialen und politischen Widersprüchen erwachsen. Anders als der Mythos von Arminius spiegelt der von Vercingétorix diese Widersprüche, allein schon deshalb, weil man darüber streitet, wie die Niederlage des Galliers zu bewerten ist: Steht die Gründung Frankreichs letztlich im Zeichen der römischen Zivilisation oder ist sie Ausdruck gallischen Widerstandsgeistes ${ }^{28}$ ?

Im Anschluss an die deutsche Auffassung von Kultur entsteht dann im 19. Jahrhundert, insbesondere an dessen Ende, in Deutschland die Anschauung von der ,Kulturnation‘. Da Deutschland als ,verspätete Nation“ erst gegen Ende des 19. Jahrhunderts zur Bildung eines Nationalstaats gelangt, wird der Gedanke verbreitet, dass eine Nation auch auf der Basis der Kultur existieren könne, ohne zuvörderst nationalstaatliche Strukturen herausgebildet $\mathrm{zu}$ haben $^{29}$. Zugleich dient die Besinnung auf die ,Kulturnation“ als Gegenargument zur vor allem in Frankreich verbreiteten These von den ,deux Allemagnes': dem vor allem unter Bismarck - militaristisch geprägten, auf Machpolitik setzenden preußischen Staat einerseits und dem durch den deutschen Idealismus und die Romantik geprägten Kulturland ,Deutschland“ andrerseits. Da Deutschland anders als Frankreich stark regional strukturiert und bis dahin nur in geringem Maße zentralistischen Prozessen ausgesetzt ist, ist die Rede von der Kulturnation mit ihren die Gemeinschaft verbindenden Werten und Normen abgrenzend, zumal eine Kultur eher auf eine bestimmte geographische Region ausgerichtet ist als die Regionen übergreifende Zivilisation. Mit der Vorstellung von der ,Kulturnation“ ist zugleich eine mögliche Verengung des Begriffs ,Staatsvolk‘ auf die ,Abstammungsgemeinschaft‘ als Basis der Nation gegeben, wie sie später im Nationalsozialismus praktiziert wird.

$28 \mathrm{Zu}$ diesen Unterschieden vgl. Charlotte Tacke, Denkmal im sozialen Raum. Nationale Symbole in Deutschland und Frankreich im 19. Jahrhundert (Kritische Studien zur Geschichtswissenschaft. 108), Göttingen, Vandenhoeck \& Ruprecht, 1995, S. 29-44.

29 So der Kulturhistoriker Friedrich Meinecke, der neben dem Kulturbesitz eines Volkes vor allem religiöse Gemeinsamkeiten als verbindendes kulturelles Element der Nation ausmacht (Vgl. dazu Georg Schmidt, „Friedrich Meineckes Kulturnation. Zum historischen Kontext nationaler Ideen in Weimar-Jena um 1800“, Historische Zeitschrift Bd. 284/2007, S. 597-622). 


\subsection{Zivilisation und Kultur nach 1945}

In der Zeit nach dem zweiten Weltkrieg wird die antithetische Verwendung der Begriffe ,Zivilisation` vs. ,Kultur` in den beiden Nationen allerdings zunehmend obsolet. Mit dem Ende dieses Krieges, der in Europa nahezu alle Gewissheiten zum Einsturz bringt, wird auch die Phase der Dekolonialisierung eingeleitet, die die Anschauung einer Überlegenheit der - westlichen - Zivilisation ins Wanken bringt. Spätestens mit der postmodernen Wende in der zweiten Hälfte des 20. Jahrhunderts, die mit den ,postcolonial studies‘ auch die theoretische Aufarbeitung des Kolonialismus mit sich bringt, ist es kaum mehr möglich, von einer universalistischen ,Zivilisation“ zu sprechen. Samuel Huntington unterscheidet in seinem Buch vom Clash of Civilisations (1996) ${ }^{30}$ denn auch weltweit acht Kulturräume bzw. Zivilisationen, die in einer globalisierten Welt ihre Interessenkonflikte untereinander austragen. Mit dem in den Sechzigerjahren gewachsenen Misstrauen gegenüber allen ,master narratives ' und der Anerkennung weltweit hybrider Formen unterschiedlicher Zivilisationen ist die Rede von ,der Zivilisation', zumal der europäischen, die in zahlreichen ehemaligen Kolonien als Herrschaftsdiskurs empfunden wird, hinfällig. So weicht denn in der heftig geführten aktuellen Debatte die Erzählung von der ,grandeur' Frankreichs und seiner Zivilisation zunehmend der Erkenntnis einer ,identité malheureuse ${ }^{\text {311 }}$, die die Gesellschaft des Landes als postnationale und multikulturelle ohne klare Vorstellungen von ihrer Identität geschweige denn ,grandeur - beschreibt.

Ganz ähnlich ergeht es dem Verständnis von Kultur seit der Nachkriegszeit. Da Deutschlands kultureller Sonderweg - dokumentiert durch die Gräuel der Nazizeit - offenkundig gescheitert war, gab es nur eine Perspektive: aktiv am Aufbau einer neuen, gemeinsamen europäischen Ordnung zu arbeiten. Durch die neu gebildete Achse Deutschland-Frankreich und die seitdem beschworene deutsch-französische Freundschaft wird die Unterscheidung Kultur und Zivilisation irrelevant, zumal beide Kategorien genau genommen schon immer ähnliche Anschauungen beinhalteten und in Frankreich (und auch in England) ohnehin nie strikt voneinander getrennt wurden. Im Zuge der Globalisierung gegen Ende des 20. Jahrhunderts und der Beeinflussung der unterschiedlichen Kulturen durch

30 Der Titel wird angesichts der zunehmenden Irrelevanz der Unterscheidung von Zivilisation und Kultur bezeichnenderweise mit „Der Kampf der Kulturen” wiedergegeben.

31 Vgl. das entsprechende Buch von Alain Finkielkraut, L'Identité malheureuse, Paris, Stock, 2013. Vgl. z. B. auch die Debatte zwischen Donald Morrison und Antoine Compagnon, Que reste-t-il de la culture française? suivi de Le Souci de la grandeur, Paris, Denoël, 2008; auf Englisch: The Death of French Culture, Cambridge/Malden, MA., Polity Press, 2010, und viele weitere Beispiele. 
Einflüsse des Internet gewinnt auch die Annäherung der französischen und der deutschen Kultur an Geschwindigkeit. Immer wieder kommen Debatten um eine sogenannte ,Leitkultur‘ auf, die in Zeiten des Multikulturalismus benötigt würde, um „gewachsene Identitäten“ von Gemeinschaften und Nationen zu bewahren ${ }^{32}$. Solche Identitäten kann man im Sinne der überlieferten deutschen Anschauung von der ,Kultur` als ethnische und damit exklusive Identität fassen oder im Sinne des französischen Zivilisationskonzepts als rechtspolitische, verkörpert durch den „citoyen“. Die Idee der Leitkultur geht zurück auf Montesquieus 1748 verfasste Schrift De l'esprit des lois, die versucht, den allgemeinen Geist einer Nation (,esprit général') aus Faktoren wie dem Klima, den Sitten, der Religion und dem Handel abzuleiten, um so das jeweilige Regierungs- und Rechtssystem zu erklären ${ }^{33}$. Die Idee, auch in einem ,kulturell vielfältigen [Gemeinwesen] einen Konsens über Werte und Normen als eine Art innere Hausordnung“34 herzustellen, stößt in Deutschland auf die nachkriegsbedingte grundsätzlich mangelnde Herausbildung eines affektiven Nationalbewusstseins. In Zeiten der Globalisierung ist es überdies schwierig zu definieren, woraus eine Leitkultur bestehen sollte, zumal Hierarchisierungen im Bereich der Kultur vom Verdikt postmoderner Ablehnung leitender Narrative getroffen werden. Somit ist auch der Kulturbegriff wie der der Zivilisation den Pluralisierungs- und Hybridisierungstendenzen der Gegenwart ausgesetzt.

Kultur kann nicht als normativ wertend angesehen werden, wonach Hochkulturen von niedrigeren kulturellen Formen unterschieden werden. Eine einseitige Beschäftigung mit Hochkulturen ist ebenso problematisch, wie die von einem großen Teil der anglo-amerikanischen ,Cultural Studies‘ betriebene Konzentration auf Alltags- und Populärkulturen, die durch ein normatives Kulturverständnis lange Zeit ins Abseits gedrängt wurden. Ebenfalls problematisch erscheint die systemtheoretische Beschränkung auf ein innerhalb der nach funktionalen Systemen ausdifferenzierten Gesellschaft vermeintliches Subsystem einer eigentlichen Kultur bestehend aus Kunst, Bildung und Wissenschaft. Kultur wird somit heute weitgehend verstanden als ein Symbolsystem, in dem sich die Anschauungsformen, affektiven Haltungen und Wertvorstellungen einer Gemeinschaft

32 Vgl. z. B. Bassam Tibi, „Leitkultur als Wertekonsens. Bilanz einer missglückten deutschen Debatte“, Aus Politik und Zeitgeschehen Bd. 1-2/2001, S. 23-26, hier: S. 23; online: http://www.bpb. de/apuz/26535/leitkultur-als-wertekonsens.

33 Vgl. den Untertitel der Schrift: „Ou du rapport que les loix doivent avoir avec la constitution de chaque gouvernement, les mœurs, le climat, la religion, le commerce \&c., à quoi l'Auteur a ajouté des recherches nouvelles sur les Loix Romaines touchant les Successions, sur les Loix Françoises et sur les Loix Féodales”.

34 Tibi, „Leitkultur als Wertekonsens“, S. 24. 
äußern. Aus der Perspektive einer kritischen Kulturwissenschaft, für die der Romanist Paul Geyer plädiert, gilt es im Folgenden, für Deutsche und Franzosen typische Deutungsmuster und Handlungsorientierungen im Hinblick auf die dahinter stehenden Wertbegriffe sowie Interessen kritisch zu hinterfragen ${ }^{35}$. Dazu ist vor allem sinnvoll, zunächst einmal die mentalen Einstellungen, die sich im Laufe der Geschichte herausgebildet haben, historisch nachzuvollziehen. Vor allem gilt es - wie am Beispiel der französischen Einstellung zur ,grandeur - nach der langen historischen Debatte um die Antithese ,Zivilisation` vs. ,Kultur` zu zeigen, inwieweit bestimmte Einstellungen immer noch virulent sind. Und dies gilt vor allem für die unterschiedlichen Kommunikationsstile in Frankreich und in Deutschland.

35 „On the Dialectics of Culture and Civilization in Critical Cultural Studies”; Vortrag bei der Tagung: Global Concepts? Keywords and their Histories, veröffentlicht vom Excellenz-Cluster Asia and Europe in a Global Context der Universität Heidelberg am 28.10.2010, S. 7; online: https:// www.romanistik.uni-bonn.de/bonner-romanistik/personal/geyer/schriften/dialectics.pdf. 